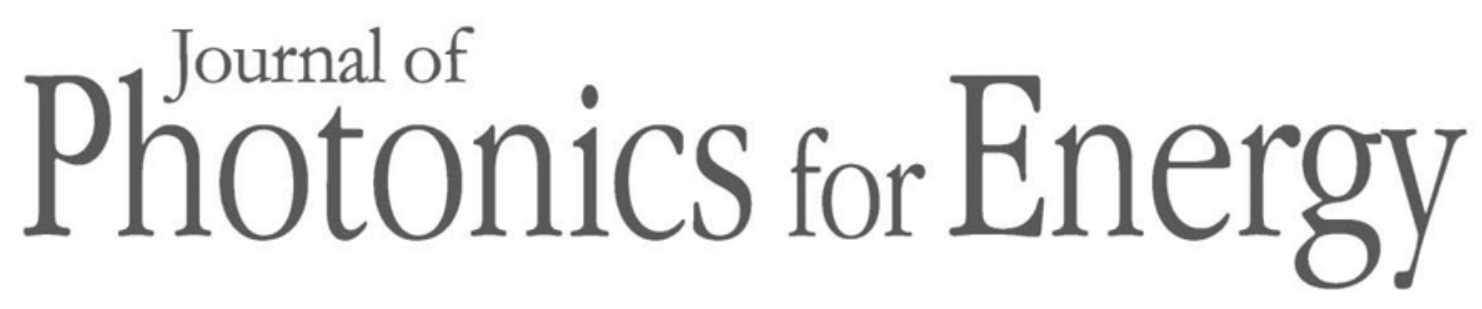

PhotonicsforEnergy.SPIEDigitalLibrary.org

\title{
Nano- and microlenses as concepts for enhanced performance of solar cells
}

Martina Schmid

Phillip Manley 


\title{
Nano- and microlenses as concepts for enhanced performance of solar cells
}

\author{
Martina Schmid $^{\mathrm{a}, \mathrm{b}, *}$ and Phillip Manley ${ }^{\mathrm{a}}$ \\ ${ }^{a}$ Helmholtz-Zentrum Berlin, Department of Renewable Energy, Nanooptical Concepts for PV, \\ Hahn-Meitner-Platz 1, 14109 Berlin, Germany \\ ${ }^{\mathrm{b}}$ Freie Universität Berlin, Department of Physics, Arnimallee 14, 14195 Berlin, Germany
}

\begin{abstract}
Both metallic nanoparticles exhibiting plasmonic effects and dielectric nanoparticles coupling the light into resonant modes have shown successful applications to photovoltaics. On a larger scale, microconcentrator optics promise to enhance solar cell efficiency and to reduce material consumption. Here, we want to create a link between the concentrators on the nano- and on the microscale. From metallic nanospheres, we turn to dielectric ones and then look at increasing radii to approach the microscale. The lenses are investigated with respect to their interaction with light using three-dimensional simulations with the finite-element method. Resulting maps of local electric field distributions reveal the focusing behavior of the dielectric spheres. For larger lens sizes, ray tracing calculations, which give ray distributions in agreement with electric field intensities, can be applied. Calculations of back focal lengths in geometrical optics coincide with ray tracing results and allow insight into how the focal length can be tuned as a function of particle size, substrate refractive index, and the shape of the microlens. Despite the similarities we find for the nano- and the microlenses, integration into solar cells needs to be carefully adjusted, depending on the goals of material saving, concentration level, focal distance, and lens size. (C) 2015 Society of Photo-Optical Instrumentation Engineers (SPIE) [DOI: 10.1117/1.JPE.5.057003]
\end{abstract}

Keywords: light coupling; plasmonics; nanosphere; microlens; concentration; focal length; ray tracing; solar cell.

Paper 14043SSP received Jul. 18, 2014; revised manuscript received Oct. 10, 2014; accepted for publication Oct. 15, 2014; published online Nov. 25, 2014.

\section{Introduction}

Plasmonic absorption enhancement by metal nanoparticles has been identified as a promising concept for boosting solar cell efficiency. ${ }^{1}$ High angle scattering, enhanced near-fields and, in the case of regular particle arrangements, coupling into guided modes are the main mechanisms identified to improve light absorption. ${ }^{2}$ As a result, plasmonic nanoparticles have been studied for various types of solar cells, such as silicon, ${ }^{3}$ organic, ${ }^{4}$ dye-sensitized, GaAs, and recently chalcopyrite-based ones. ${ }^{5}$ Another approach for light trapping, dielectric nanoparticles, is a result of the search for increased efficiency, but also offers an easy and stable integration into the solar cell. Related effects of light coupling can be attributed to whispering gallery modes, ${ }^{6}$ but general anti-reflection effects also play a fundamental role. ${ }^{7}$ Compared to their metallic counterparts, dielectric nanoparticles may show equally strong scattering behavior, yet without any related absorption losses. However, they may lose in terms of high angle scattering, yet exhibit a strong forward scattering. ${ }^{8}$ First, we will briefly revisit near-fields around metallic and dielectric nanospheres, and then turn to dielectric spheres of increasing size which show a progressive trend toward a defined focal spot.

Micrometer-sized dielectric spheres have been identified to give rise to so-called photonic nanojets, which were identified early to have the potential for a novel ultramicroscopy technique. ${ }^{9}$ Detailed studies of the nanojet evolution as a function of parameters such as wavelength,

*Address all correspondence to: Martina Schmid, E-mail: martina.schmid@helmholtz-berlin.de

0091-3286/2015/\$25.00 (C) 2015 SPIE

Journal of Photonics for Energy

057003-1

Vol. 5, 2015 
particle size and refractive index of sphere, and surrounding have followed. ${ }^{10,11}$ In this paper, we want to focus on the similarity of light concentration behind dielectric particles and the well-known focusing behavior of lenses. We will show how the focus of the nanojets can be equally described by ray tracing methods as well as in geometrical optics calculations for light propagation through a lens for the case of microspheres large compared to the wavelength. The transition to plano-convex lenses will be highlighted since this relates to the particular application in solar cells which has recently attracted attention: microconcentrator solar cells. In this concept, the sunlight is concentrated by micro-optics onto micrometer-sized solar cells for the purpose of material saving and efficiency enhancement. ${ }^{12,13}$ This reduces the well-known concept of concentrator photovoltaics ${ }^{14}$ to the micrometer scale in order to fabricate compact high efficiency devices. Since commonly available lens arrays are often characterized by segment-like shapes, we will address shape effects as well as the influence of the substrate's refractive index.

By establishing the link between the nanoscaled metallic and dielectric particle concentrators on one hand, and the lens-like microconcentrators on the other hand, we aim at providing a unified picture of the different aspects of light concentration for efficiency enhancement, particularly in photovoltaic devices. These two different aspects may be classified as follows: (1) Absorption enhancement by locally enhanced near-fields, preferential forward and high angle scattering for improved incoupling and prolonged optical path lengths. This aspect will lead to an increased short-circuit current density $j_{\mathrm{sc}}$ with an upper limit given by $j_{\mathrm{sc}, \max }=\int_{0}^{\lambda_{g}} \frac{\mathrm{AM} 1.5(\lambda) \cdot \lambda}{h \cdot c} \mathrm{~d} \lambda$. (2) Increased photon flux density by concentrating the incoming light from an area $A_{0}$ to $A_{\text {cell }}$ with a concentration factor of $C=A_{0} / A_{\text {cell }}$. This effect can lead to a maximum short-circuit current density $j_{\mathrm{sc}, \max }=\int_{0}^{\lambda_{g}} C \cdot \frac{\mathrm{AM} 1.5(\lambda) \cdot \lambda}{h \cdot c} \mathrm{~d} \lambda$, and the open-circuit voltage $V_{\mathrm{oc}}$ is enhanced according to $V_{\mathrm{oc}} \propto \ln \left(\frac{C \cdot j_{\mathrm{sc}}}{j_{0}}\right) .{ }^{15}$ Going from (1) to (2) requires a reduced device area for maximum benefit and we will find the lens sizes where this becomes relevant.

\section{Approach and Computational Methods}

The modification of light propagation by nano- and micrometer-sized spheres, hemispheres, and plano-convex lenses is investigated. We start with a comparison of small metallic (Ag) versus dielectric $\left(\mathrm{SiO}_{2}\right)$ nanospheres, and then focus on the dielectric case when investigating increasing sizes and various shapes. Ag refractive index data are interpolated to $0.14221+4.52296 i$ according to the data from Ref. 16, whereas the refractive index for $\mathrm{SiO}_{2}$ is set to $n=1.53839$ (according to Ref. 17) for the wavelength $\lambda=700 \mathrm{~nm}$ investigated here. We focus on this wavelength due to its significance for the application to chalcopyrite solar cells with reduced absorber thickness, which requires an absorption enhancement in the range starting from this wavelength. Chalcopyrites, or $\mathrm{Cu}(\mathrm{In}, \mathrm{Ga}) \mathrm{Se}_{2}$ (CIGSe) compounds, are used as absorber materials in thin-film solar cells due to their direct band gap and resulting good efficiencies. But they also come with the aim of reducing material consumption since they contain the rare element indium. When thinking about the application of concentrator lenses to this type of solar cells, the refractive indices of the absorber layer, which is approximately 2.8, and that of the front contact $\mathrm{ZnO}: \mathrm{Al}$, which is approximately 1.8, are of interest. We will exemplarily use them when assigning particular refractive indices to substrates later on. The imaginary part of the refractive index will be kept to zero in order to follow the light propagation. For investigating the intensity distributions and focusing effects of the nanoand microlenses, three approaches are followed: three-dimensional (3-D) optical simulations using the finite-element method (FEM), ray tracing, and geometrical optics calculations. The FEM and ray tracing are briefly described in the following, whereas Sec. 3 is dedicated to the geometrical optics calculations. The generalized structure of the shapes can be seen in Fig. 1 as used for the geometrical optics calculations.

The 3-D optical simulations are carried out using the software package JCMsuite. ${ }^{18,19}$ It is based on the FEM, i.e., the computational domain is divided into geometrical primitives with a discretization adaptable to the particular geometry. Using local polynomial functions, the solution space to Maxwell's equations in the time-harmonic ansatz for the entire domain can be 


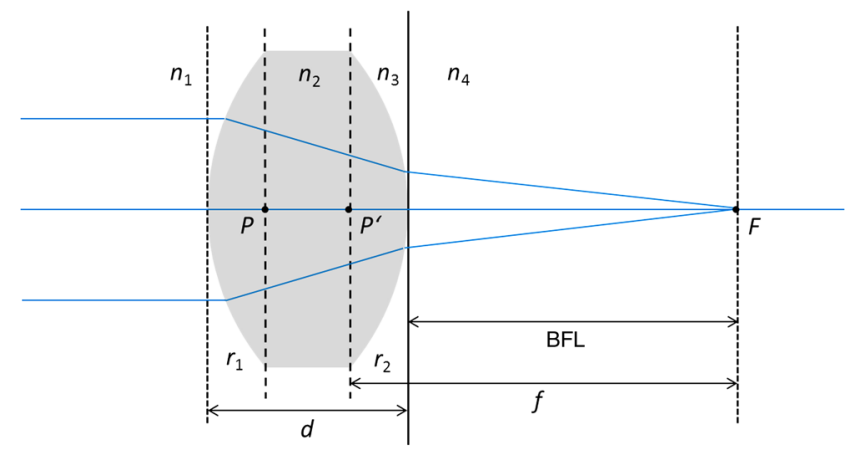

Fig. 1 Schematic of a generalized lens structure defined by two curved surfaces with radii $r_{1}$ and $r_{2}$ and a total thickness of $d$ with the lens having a refractive index of $n_{2}$ while being embedded in an incoming medium with $n_{1}$ and an outgoing medium with $n_{3}$, eventually also on top of a substrate with $n_{4}$.

approximated. In the examples presented here, the cylindrical symmetry of the geometry has been exploited to reduce the computational effort. This allows the solutions to Maxwell's equations to be expanded in a Fourier basis, which accounts for the angular dependence. ${ }^{20}$ The computational domain was terminated by perfectly matched layer (PML) boundary conditions. The total electric field surrounding the particle was calculated. Due to the memory requirements of the FEM, a computational domain size which is larger than a few tens of wavelengths becomes costly, even when exploiting the cylindrical symmetry as explained above. This meant that for our chosen wavelength of $700 \mathrm{~nm}$, calculations for particle diameters larger than 10,000 nm became very time consuming using the FEM. Accordingly, near-field pictures shown in the following represent the electric field distribution normalized to the incoming field $|E| /\left|E_{0}\right|$ displayed in the $x z$-plane at $y=0$; the $z$-direction is defined as the negative propagation direction of the incident light with polarization along the $x$-direction.

Sequential ray tracing describes the propagation of light rays when traveling from an object to an image plane via a given sequence of surfaces. At each of the surfaces, the ray propagation is described according to the laws of reflection and refraction and no paraxial approximation is made. By following the path of a ray, its angle of incidence onto an optical surface can be identified and Snell's law accordingly applied. Ray tracing is mostly used for the design of optical elements and systems and this is very suitable for our investigations of micrometer-sized lenses, where near-field effects are negligible. For the calculations shown here, we used the software Radiant Zemax, Version 13. ${ }^{21}$

\section{Geometrical Optics Calculations}

Geometrical optics or ray optics can be used to describe light propagation through media and objects much larger than the wavelength. Based on this approach, we investigate the rear focal length $f$ and the back focal length (BFL) for a generalized structure of a lens as depicted in Fig. 1. The lens is defined by two curved surfaces with radii $r_{1}$ and $r_{2}$ and a total thickness of $d$ and has a refractive index of $n_{2}$; it is embedded in an incoming medium with $n_{1}$ and an outgoing medium with $n_{3}$, eventually on top of a substrate with $n_{4}$.

Details of the derivation are given in Ref. 22. Here, we only summarize the results which are the Generalized Lensmaker's equation:

$$
\frac{1}{f}=\frac{n_{2}-n_{1}}{n_{4}} \cdot \frac{1}{r_{1}}-\frac{n_{2}-n_{3}}{n_{4}} \cdot \frac{1}{r_{2}}+\frac{\left(n_{2}-n_{1}\right) \cdot\left(n_{2}-n_{3}\right)}{n_{2} \cdot n_{4}} \cdot \frac{d}{r_{1} \cdot r_{2}},
$$

and the back focal length

$$
\mathrm{BFL}=f \cdot\left(1-\frac{n_{2}-n_{1}}{n_{2}} \cdot \frac{d}{r_{1}}\right)
$$


Table 1 Formulas for the rear focal length and the back focal length (BFL) for spheres, hemispheres, and segments in air and on a substrate according to the generalized lensmaker's equation, Eq. (1) and the equation for the BFL, Eq. (2).

\begin{tabular}{|c|c|c|}
\hline Configuration & Focal length & Back focal length \\
\hline $\begin{array}{l}\text { Sphere of radius } r=r_{1}=-r_{2} \text { with refractive } \\
\text { index } n=n_{2} \text { in air }\left(n_{1}=n_{3}=n_{4}=1\right) ; d=2 r\end{array}$ & $\frac{1}{f}=\frac{n-1}{n} \cdot \frac{2}{r}$ & $\mathrm{BFL}=f \cdot \frac{2-n}{n}=\frac{2-n}{2(n-1)} \cdot r$ \\
\hline $\begin{array}{l}\text { Sphere of radius } r \text { with refractive index } \\
n_{2}=n \text { in air }\left(n_{1}=n_{3}=1\right) \text {, but on } \\
\text { a substrate with } n_{4}=n_{\text {subs }}\end{array}$ & $\frac{1}{f}=\frac{n-1}{n \cdot n_{\text {subs }}} \cdot \frac{2}{r}$ & $\mathrm{BFL}=f \cdot \frac{2-n}{n}=\frac{(2-n) \cdot n_{\text {subs }}}{2(n-1)} \cdot r$ \\
\hline $\begin{array}{l}\text { Hemisphere of radius } r=r_{1}=d, r_{2}=\infty \\
\text { with refractive index } n=n_{2} \text { in air } \\
\left(n_{1}=n_{3}=n_{4}=1\right)\end{array}$ & $\frac{1}{f}=(n-1) \cdot \frac{1}{r}$ & $\mathrm{BFL}=f \cdot \frac{1}{n}=\frac{1}{n(n-1)} \cdot r$ \\
\hline $\begin{array}{l}\text { Hemisphere of radius } r \text { with refractive } \\
\text { index } n_{2}=n \text { in air }\left(n_{1}=n_{3}=1\right) \text {, but on } \\
\text { a substrate with } n_{4}=n_{\text {subs }}\end{array}$ & $\frac{1}{f}=\frac{n-1}{n_{\text {subs }}} \cdot \frac{1}{r}$ & $\mathrm{BFL}=f \cdot \frac{1}{n}=\frac{n_{\text {subs }}}{n(n-1)} \cdot r$ \\
\hline $\begin{array}{l}\text { Segment of width } s \text {, height } h=s \cdot \text { ratio }=d \text {, } \\
\text { radius } r \text { according to Eq. (3) and with refractive } \\
\text { index } n=n_{2} \text { in air }\left(n_{1}=n_{3}=n_{4}=1\right)\end{array}$ & $\frac{1}{f}=(n-1) \cdot \frac{1}{r}$ & $\mathrm{BFL}=f \cdot\left(1-\frac{n-1}{n} \cdot \frac{h}{r}\right)=\frac{1}{n-1} \cdot r-\frac{1}{n} \cdot h$ \\
\hline $\begin{array}{l}\text { Segment of width } s \text {, height } h=s \cdot \text { ratio, } \\
\text { radius } r \text { and with refractive index } n_{2}=n \text { in air } \\
\left(n_{1}=n_{3}=1\right) \text {, but on a substrate with } n_{4}=n_{\text {subs }}\end{array}$ & $\frac{1}{f}=\frac{n-1}{n_{\text {subs }}} \cdot \frac{1}{r}$ & $\mathrm{BFL}=f \cdot\left(1-\frac{n-1}{n} \cdot \frac{h}{r}\right)=\frac{n_{\text {subs }}}{n-1} \cdot r-\frac{n_{\text {subs }}}{n} \cdot h$ \\
\hline
\end{tabular}

The particular shapes investigated are spheres, hemispheres and circular segments in air and on a substrate. The spherical elements are described by the radius $r$ and the segments by the segment width $s$ and the height $h$, resulting in $r=\left(4 h^{2}+s^{2}\right) /(8 h)$ for the latter ones. With the definition of the height as a certain ratio of the segment width this can be expressed as

$$
r_{\text {segment }}=\frac{s \cdot\left(4 \cdot \text { ratio }^{2}+1\right)}{8 \cdot \text { ratio }} \text { for } h=\text { ratio } \cdot s
$$

The resulting equations for the rear focal length and the BFL for the various cases are summarized in Table 1.

\section{Results and Discussions}

Looking at spheres on the nanoscale, a strong near-field enhancement is one of the reasons that metallic nanoparticles are of interest for absorption enhancement. Figure 2(a) shows the normalized electric near-field distribution (calculated with FEM) around a Ag nanoparticle with $r=$ $200 \mathrm{~nm}$ at a wavelength of $700 \mathrm{~nm}$. The representation is a cut through the $x z$-plane at $y=0$ with polarization along the (horizontal) $x$-direction and the $k$-vector along the negative $z$-direction. It clearly reveals a high concentration of the field around the boundary of the metallic nanoparticle, which is due to the free charge carriers repelling fields from inside the nanoparticle. Additionally, the lobes of this quadrupole mode are visible, highlighting the second benefit of plasmonic nanoparticles, namely the reradiation of light into high angles, potentially leading to light trapping in an underlying layer. Despite these benefits, metallic nanoparticles may also show parasitic absorption resulting in losses in the form of heat. This disadvantage together with the challenge of stable integration into solar cells ${ }^{23}$ leads to the consideration of nanoparticles made from dielectric materials, which can equally show high scattering ability with similar resonance wavelengths for slightly larger sizes. A detailed comparison of metallic versus dielectric nanoparticles was conducted in Ref. 8. Here, we focus on the very particular electric field distribution around dielectric nanoparticles as shown in Fig. 2(b) for the example of a $\mathrm{SiO}_{2}$ nanosphere with $r=300 \mathrm{~nm}$. The light is preferentially guided to the forward direction and concentrated in the shadow region behind the nanoparticle. This focusing effect, already 


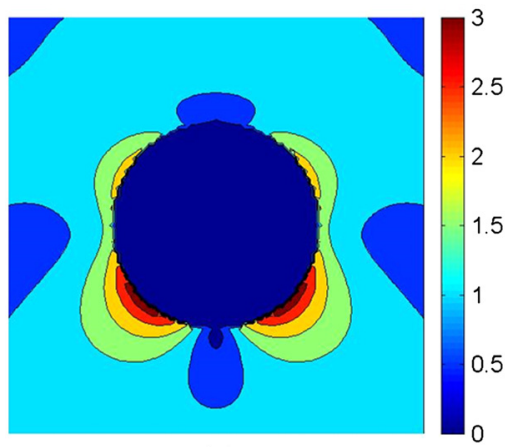

(a)

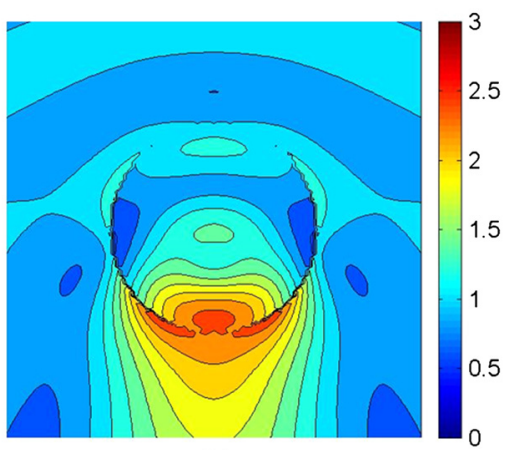

(b)

Fig. 2 Electric near-field distribution around a metallic versus a dielectric nanoparticle: (a) $\mathrm{Ag}$ nanoparticle with radius $200 \mathrm{~nm}$, (b) $\mathrm{SiO}_{2}$ nanoparticle with radius $300 \mathrm{~nm}$ in air; depicted is the electric field in the $x z$-plane normalized to the incident field with polarization along the $x$-direction and $k$-vector along the negative $z$-direction at a wavelength of $700 \mathrm{~nm}$.

observable for dielectric particles smaller than the wavelength, will be a center point of our discussion as we proceed to larger sphere sizes.

Figure 3 reveals how the point of the maximum field shifts away from the sphere with increasing radius. Figures 3(a) to 3(c) show the electric field distribution around $\mathrm{SiO}_{2}$ dielectric spheres with radii of 1,2 , and $3 \mu \mathrm{m}$, respectively. The maximum of the field, just being on the border of the sphere for the case of $r=1 \mu \mathrm{m}$, gradually moves outward and approaches the back focal point as calculated from geometrical optics (Table 1) and marked by the cross. Figure 3(d) gives the dependency of the point of maximum intensity (PMI) normalized to the respective radius as a function of $r$. For radii smaller than $1 \mu \mathrm{m}$ it is negative which means the PMI is inside the sphere. For increasing radii, the PMI increases and starts to saturate. A linear dependency of the back focal point on the radius is expected in geometrical optics and was also calculated in the 3-D simulations e.g., for microcylinders by Ref. 24. From the $r$-range simulated here, a PMI with a maximum of 0.19 can be fitted, whereas the geometrical optics calculation gives 0.43 . The saturation appears to not be fully reached yet, but another fact also comes into play, namely that the maximum intensity resulting from calculations of electric field distributions was, in our simulations, always observed to stay below the value of the geometrical back focal point. We attribute this to the particular intensity distribution in the high intensity region as described by Ref. 11 . The profile of optical intensity of the nanojet in the direction of propagation is asymmetric with the maximum at shorter distances. Thus, the $z$-value attributed to the center of the high intensity region deviates from the $z$-value of the absolute maximum.

However, when comparing the light distribution resulting from ray tracing simulations to the electric field distributions extracted from simulations with the 3-D FEM, we find a good overall agreement for spheres with $r=2.5 \mu \mathrm{m}$. Figures 4(a) to 4(c) show a $\mathrm{SiO}_{2}$ sphere in air and on

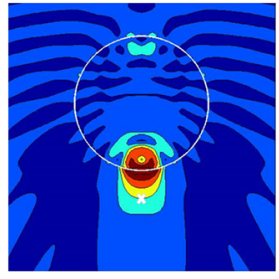

(a)

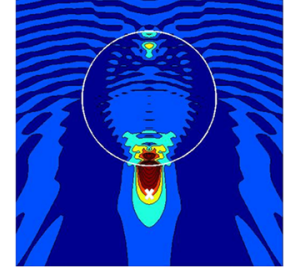

(b)

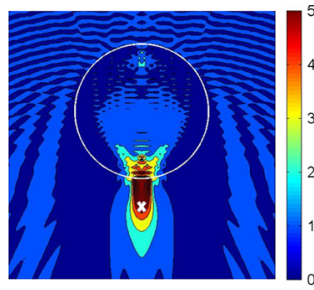

(c)

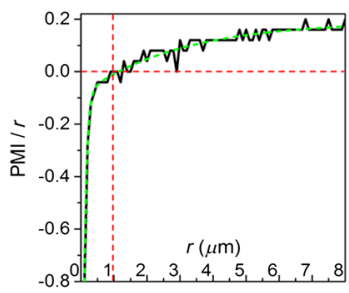

(d)

Fig. 3 Normalized electric field distribution at $\lambda=700 \mathrm{~nm}$ around a $\mathrm{SiO}_{2}$ nanoparticle with increasing radius $r$ of (a) 1, (b) 2 and (c) $3 \mu \mathrm{m}$; the shape of the sphere is marked for clarity as well as the back focal length calculated from geometrical optics (cross); (d) gives the point of maximum intensity with respect to the sphere's rear surface normalized to its radius and plotted as a function of the radius. 


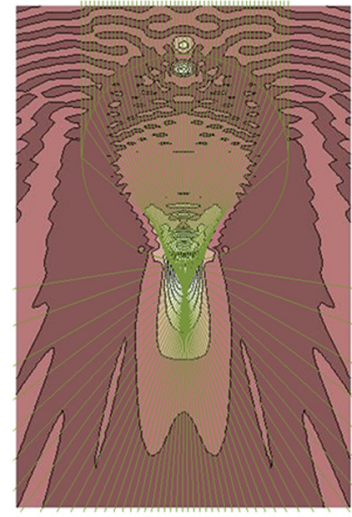

(a)

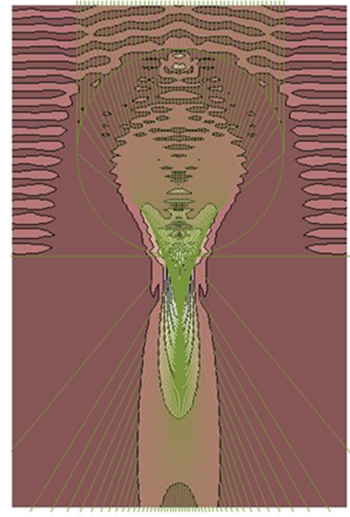

(b)

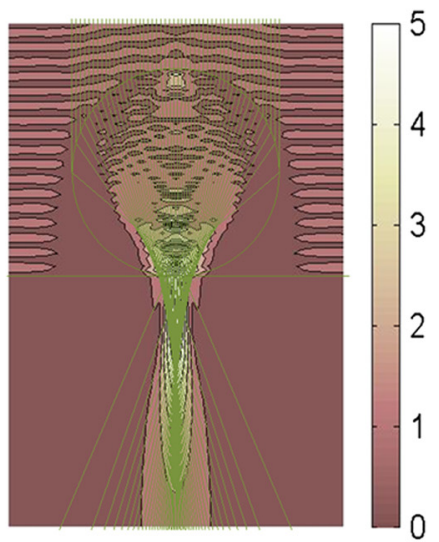

(c)

Fig. 4 Focus of a $\mathrm{SiO}_{2}$ sphere with $r=2.5 \mu \mathrm{m}$ (a) in air, (b) on an $n=1.8$ substrate, (c) on an $n=2.8$ substrate, seen in electric field distribution (brown/tan) and ray tracing (green).

a substrate with a constant refractive index $n=1.8$ or $n=2.8$, respectively, which can be attributed to the deposition on top of the front $\mathrm{ZnO}: \mathrm{Al}$ contact or on top of the CIGSe absorber when correlating to the application in solar cells. An absorption coefficient of zero was chosen for the substrate layers to fully follow the ray propagation without any absorption losses. The ray propagation is plotted as an overlay to the electric field distribution, which reveals that the focus of the rays falls well into the area of highest field enhancement. With an increasing substrate refractive index, the focus shifts away from the sphere, showing us that the choice of an adequate substrate material is one option with which to tune the PMI or the back focal length.

Another way of tuning the focal length is by changing the shape of the lens. By moving from spherical to hemispherical and from this further to segment-shaped lenses, we can also increase the BFL. The diameter $d$ which becomes the segment width $s$ is kept constant while reducing the segment height $h$. This leads to the radius of curvature of the lens increasing as $h$ decreases, which can be seen in Fig. 5. For clarity, only the ray tracing results are shown. By flattening the plano-convex lens in this way, the BFL can be increased from $0.60 \cdot d$ for a hemispherical $\mathrm{SiO}_{2}$ lens to $0.86 \cdot s$ for an $h=0.30 \cdot s$ segment and further to $2.53 \cdot s$ for an $h=0.10 \cdot s$ segment.

Figure 6 summarizes the BFLs as calculated by the geometrical optics approach (for equations, see Table 1) and which agree within deviations of $\leq 0.3 \%$ with the results from ray tracing simulations (Zemax). As given by the respective equations in Table 1, the BFL linearly increases with the substrate refractive index, see Fig. 6(a) for $\mathrm{S} \mathrm{SiO}_{2}$ sphere in air and on a substrate with an increasing refractive index $n_{\text {subs }}$. The values for the cases represented in Fig. 4 are marked with a cross. According to the formula in Table 1 and considering Eq. (3) for segment-shaped lenses, the BFL is even more sensitive to the shape, i.e., here changes in the relative height $h$ represented by the variable ratio $=h / s$, where $s$ is the segment width. Figure 6(b) shows the BFL as a function of $s / h$ for $\mathrm{SiO}_{2}$ segments in air, which reveals an almost linear dependency, especially for larger values (smaller heights). This means, the $1 / h$ term is dominating, making the changes of relative height to an approach much more sensitive for adaptation of the BFL compared to variations of the substrate refractive index, where the BFL is only linear with $n_{\text {subs }}$. Comparing the results presented here, we can conclude that both the substrate refractive index and the lens shape can have a marked impact on the BFL. However, as Fig. 6 shows, the shaping of the lens offers more scope for change in the value of BFL, especially when considering that the substrate composition may need to be optimized for electronic rather than optical efficiency.

Both an increased substrate refractive index and a reduced lens height essentially shift the focal point further from the lens. This may not be desirable for our application to solar cells where we are aiming for compact devices. Yet, in the end, the combined thickness of the lenses and BFL will determine the effective thickness of the optical elements. For direct application on top of the device, spherical microlenses appears to be more suitable, whereas with the introduction of a spacer layer of a low refractive index, other lens geometries may be preferable. Looking 


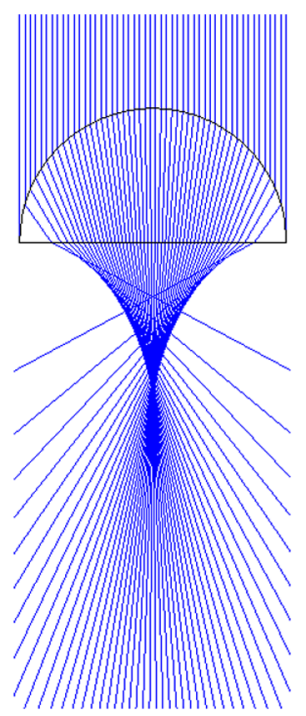

(a)

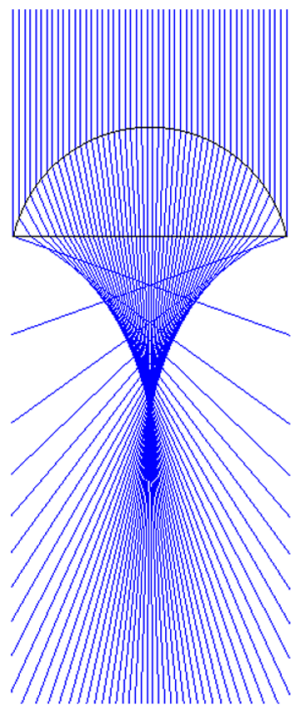

(b)

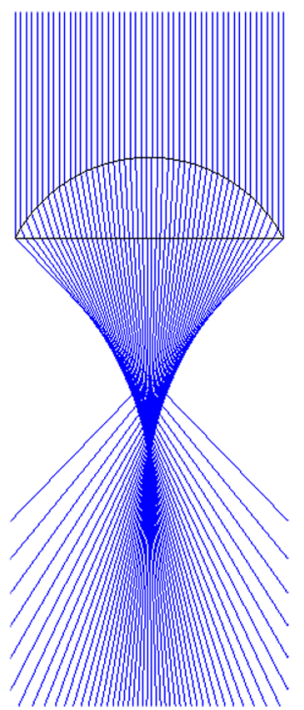

(c)

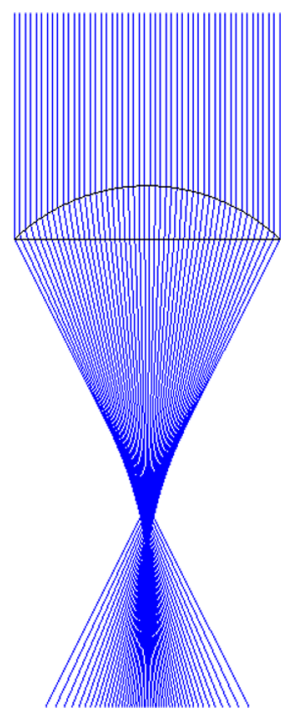

(d)

Fig. 5 Increasing back focal length of plano-convex lens made from $\mathrm{SiO}_{2}$ with decreasing height and increasing radius of curvature. The height is given as a ratio of the segment width with ratio = (a) 0.5 (hemisphere), (b) 0.4 , (c) 0.3 and (d) 0.2 .

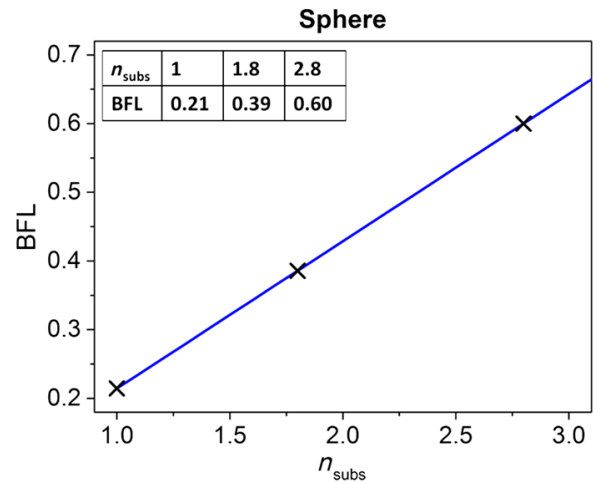

(a)

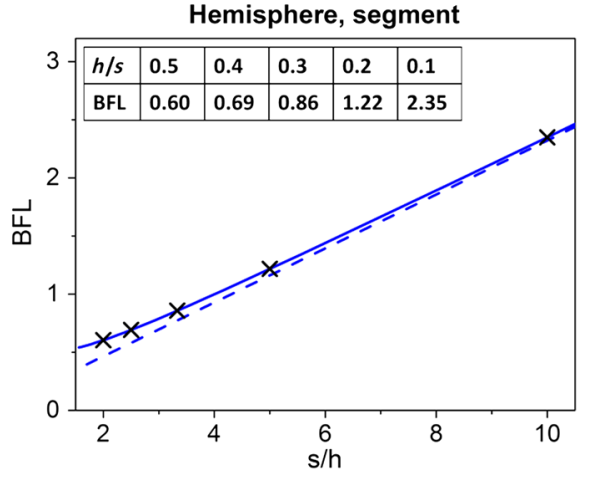

(b)

Fig. 6 Back focal length (according to Table 1 for geometrical optics calculations which agree with results from ray tracing simulations) for (a) a $\mathrm{SiO}_{2}$ sphere in air and on a substrate and (b) $\mathrm{SiO}_{2}$ hemispheres and segments in air; the total curve progressions are shown with marks for the particular cases represented in Figs. 4 and 5 ( $s$ or $2 \cdot r$ set to 1$)$.

at the influence of overall lens size, even for a substrate index of $n=1.5$, i.e., glass, and a spherical lens made from $\mathrm{SiO}_{2}$, the $\mathrm{BFL}$ in the case of a $500-\mu \mathrm{m}$ diameter lens is $\mathrm{BFL}=320 \mu \mathrm{m}$. For a 500 times smaller $\mathrm{SiO}_{2}$ spherical lens with $d=1 \mu \mathrm{m}$, the BFL in an $n=$ 1.5 substrate layer was only $\mathrm{BFL}=0.64 \mu \mathrm{m}$. Therefore, for spherical lenses, additional spacer layers may also be desirable with increasing lens size.

For a detailed analysis of light concentration as a function of sphere sizes, we go back to the local distributions of the electric field intensities. Figure 7 compares the horizontal intensity profiles $\left(|E| /\left|E_{0}\right|\right)^{2}$ at $y=0$ and a distance $z=0$, i.e., at the interface between air and an $n=$ 1.8 material on top of which $\mathrm{SiO}_{2}$ spheres with different radii are located. Behind the $r=0.3 \mu \mathrm{m}$ sphere, a broad intensity distribution over the whole lens area is found (black curve), which already shows an increase compared with the basic transmittance expected at the interface without spheres (dashed orange line which corresponds to a transmittance of 91.8\%). The red curve for an $r=0.9 \mu \mathrm{m}$ and the green curve for an $r=2.7 \mu \mathrm{m}$ sphere show how the maximum intensity increases and the peak narrows with increasing lens radius. The diameters of the lenses from 


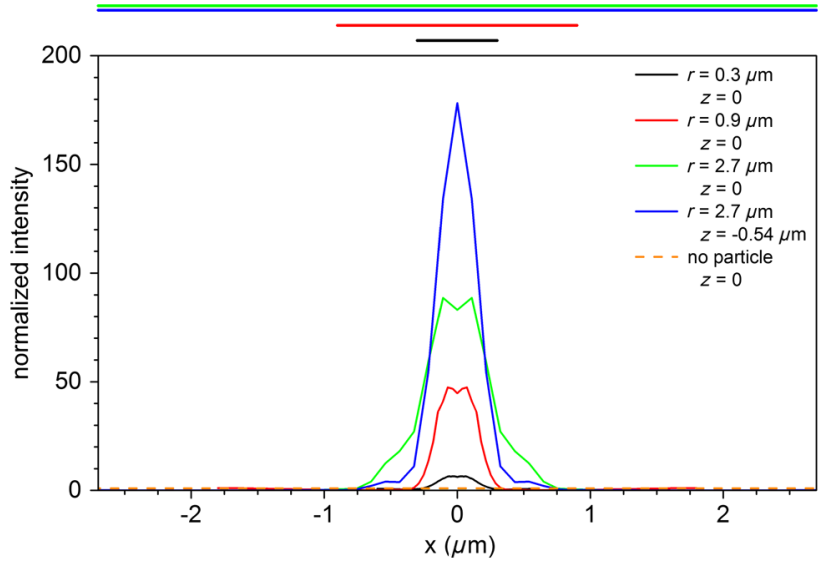

Fig. 7 Intensity profiles at $y=0$ at the interface $(z=0)$ of air and an $n=1.8$ material on top of which $\mathrm{SiO}_{2}$ spheres with sizes as given are located. The bars on the top give the diameter of the lenses from which the peaks arise (colors correspond to curves).

which the peaks arise are indicated by the bars on top of the graph (colors correspond to solid curves). The full width at half maximum for the case $r=0.9 \mu \mathrm{m}$, for which the PMI starts to be located outside the sphere, is $0.40 \cdot d$, while for a three times larger lens $(r=2.7 \mu \mathrm{m})$, it has narrowed down to $0.24 \cdot d$. The blue curve represents the line scan along the $x$-axis at $z=-0.54 \mu \mathrm{m}$, i.e., at the distance of the PMI, for the case of an $r=2.7 \mu \mathrm{m}$ sphere, which shows a further increase in intensity and a reduction in width. (Annotation: the dip at $x=0$ of the intensity profiles is attributed to a simulation artifact of the sphere touching the substrate at a singular point only; however, this does not change the trends described.)

Next, we consider a chalcopyrite solar cell placed directly beneath the three differently sized dielectric spheres. The radius of the cylindrical solar cell was set to the radius of the smallest sphere, i.e., $0.3 \mu \mathrm{m}$ and the solar cell structure was $\mathrm{ZnO}: \mathrm{Al} / \mathrm{i}-\mathrm{ZnO} / \mathrm{CdS} / \mathrm{Cu}(\mathrm{In}, \mathrm{Ga}) \mathrm{Se}_{2} / \mathrm{Mo}$ (top to bottom on glass substrate) with thicknesses 100/50/50/500/200 nm, respectively. Underneath the solar cell is a glass substrate which extended over the entire width of the domain. The simulations were carried out with the FEM and using the previously described cylindrical coordinate system. PML layers were used in all outgoing directions. For each lens radius, the amount of light absorbed in the chalcopyrite layer was calculated. The usual normalization for this absorption would be the total energy which was incident to the computational domain. However, since the system is isolated, such a normalization would cause the absorption to be dependent on the domain size. To try and avoid this dependence, we have normalized the amount of energy absorbed by the chalcopyrite layer to the amount of energy incident to either the lens or to the solar cell in the ray optics regime. The value of the absorption normalized to the lens area, $A_{L}$, is $2.42,1.11$, and 0.80 for the $0.3,0.9$, and $2.7 \mu$ m radius lens, respectively. Because we have normalized the amount of energy directly incident to the lens, the first two values are larger than 1.0. By looking at the Poynting flux lines in Figs. 8(a) and 8(b), we can see that flux lines which begin outside of the radius of the lens penetrate into the chalcopyrite layer, meaning that more light may be absorbed than was directly incident to the lens. For the largest lens, only energy flux lines which were originally incident to the lens penetrate the chalcopyrite layer. This means that for sub-wavelength-sized lenses, the lenses would not need to be closely packed to ensure the maximum absorption in the solar cell. For all lens sizes, the solar cell suffers from some parasitic absorption in the window, buffer, and back contact layer. Further, the largest lens suffers from imperfect focusing, which may be improved by positioning the solar cell so that the chalcopyrite layer is at the focal position.

If we look now at the absorption normalized to the solar cell area, $A_{\mathrm{SC}}$, the values are 2.42 , 10.01 , and 64.40 for the $0.3,0.9$, and $2.7 \mu \mathrm{m}$ radius lenses, respectively, see the comparison in Table 2. This factor relates to the concentration of light. If the entire energy incident to the lens was absorbed in the chalcopyrite layer, then the ratio of the lens and solar cell area would give us the absorbed energy, which for the sizes presented would be 1,9 , and 81 . The smaller two lenses 


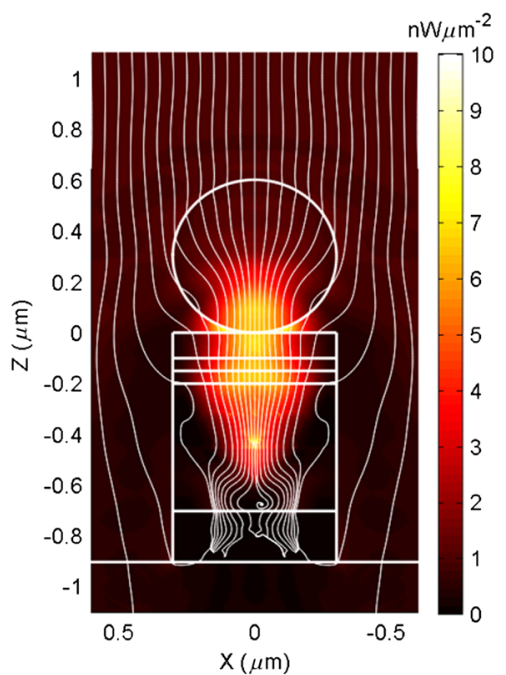

(a)

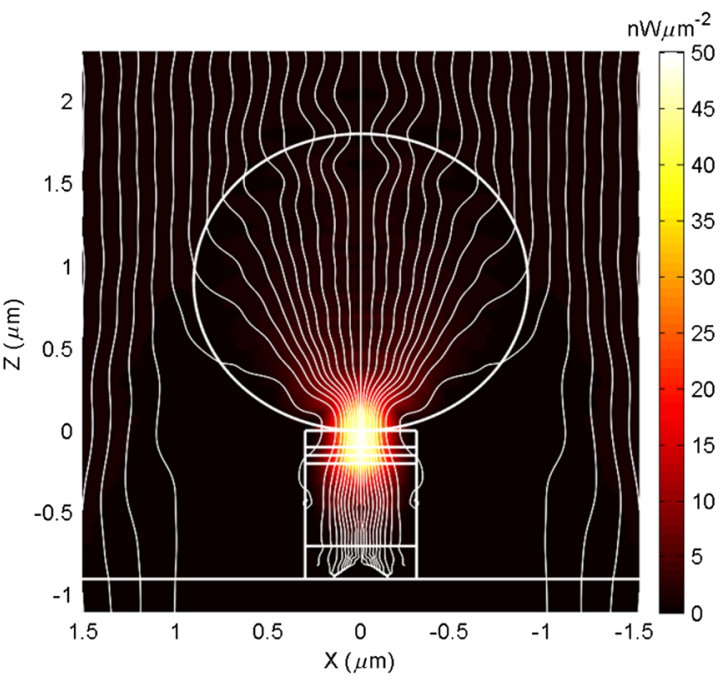

(b)

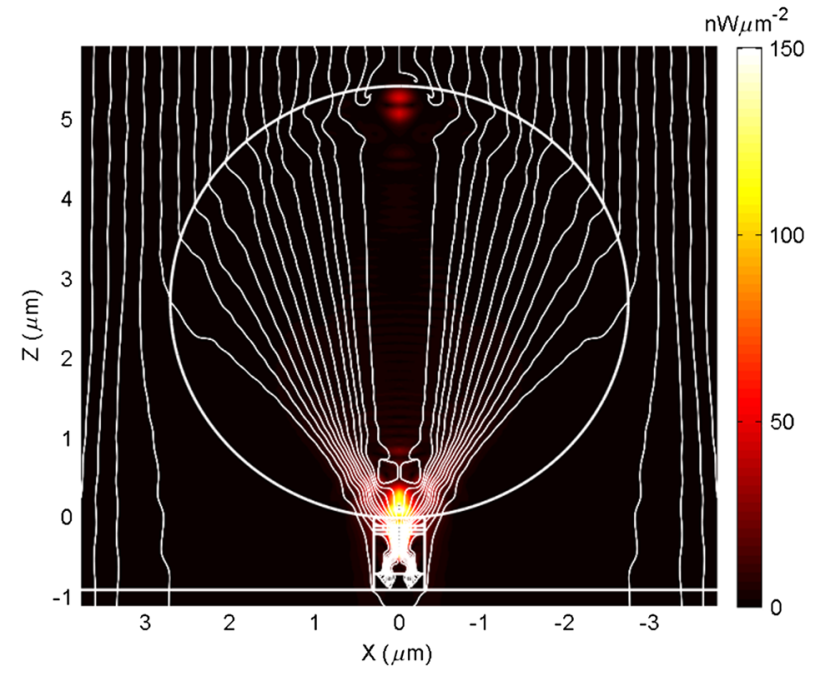

(c)

Fig. 8 Magnitude and flux lines of the Poynting vector for three differently sized lenses with radii (a) 0.3 , (b) 0.9 and (c) $2.7 \mu \mathrm{m}$ placed on a cylindrical chalcopyrite solar cell of radius $0.3 \mu \mathrm{m}$.

show a higher absorption than this simple analysis, reiterating the focusing of light which is not incident to the lens. However, it should be noted that for large material savings, while the largest lens does not offer the highest absorption per unit concentrator device area, it does show the highest absorption per unit solar cell area. This means that for reduced consumption of material in the solar cell, the largest lenses may be the most beneficial, especially if steps are taken to place the chalcopyrite layer in the focus of the beam and to ensure that it is as wide as the focal spot.

Table 2 Absorptance in the chalcopyrite layer of a cylindrical solar cell of radius $0.3 \mu \mathrm{m}$, underneath lenses of different radii normalized to either the lens area or to the solar cell area and comparison to the theoretical concentration ratio.

\begin{tabular}{lccc}
\hline \hline Lens radius $r(\mu \mathrm{m})$ & 0.3 & 0.9 & 2.7 \\
Absorption normalized to lens area $A_{L}$ & 2.42 & 1.11 & 0.80 \\
Absorption normalized to solar cell area $A_{S C}$ & 2.42 & 10.01 & 64.40 \\
Theoretical concentration ratio & 1 & 9 & 81 \\
\hline \hline
\end{tabular}




\section{Conclusions}

From metallic nanospheres, we have made the link to lossless dielectric ones and taken a detailed look at their focusing behavior particularly as a function of size. The 3-D optical simulations using the FEM reveal the strong local field enhancement in the shadow region behind the dielectric sphere and highlight the transition from near- to far-field focusing. The quantitative maps of electric field strength give insight into the local distribution of intensities including the area of maximum light concentration. For micrometer-sized spheres, this focusing area coincides with results from ray tracing which give the value of the BFL itself. The results from ray tracing are in agreement with focal lengths calculated according to the geometrical optics and give us indications of how the focal lengths can be tuned apart from adjusting the particle size. Both an increased substrate refractive index and the transition from a spherical to a hemispherical or segment-like shape can increase the focal length. For the particular application to solar cells with the aim of a compact device design, a short distance of the point of highest intensity from the concentrating lens may be required. This brings us back to spherically shaped particles of smaller sizes, which may win in terms of field enhancement in the close vicinity of the lens, and improve devices with very thin absorber layers. With respect to substantial material saving by horizontal reduction of the absorber size, however, microconcentrator solar cells may be beneficial. They rely on microscaled lenses and can be promising when integrating adequate spacer layers in order to bring the microcells into the region of high field intensities.

\section{Acknowledgments}

The authors are grateful to the Helmholtz Association for support from the Initiative and Networking Fund for the Young Investigator Group VH-NG-928.

\section{References}

1. K. R. Catchpole and A. Polman, "Plasmonic solar cells," Opt. Express 16, 21793-21800 (2008).

2. H. A. Atwater and A. Polman, "Plasmonics for improved photovoltaic devices," Nat. Mater. 9, 205-213 (2010).

3. S. Pillai et al., "Surface plasmon enhanced silicon solar cells," J. Appl. Phys. 101, 093105 (2007).

4. L. Fang et al., "Efficiency enhancement in organic solar cells with extended resonance spectrum of localized surface plasmon," IEEE Photon. J. 5, 8400307 (2013).

5. M. Schmid et al., "Modeling plasmonic scattering combined with thin-film optics," Nanotechnology 22, 025204 (2011).

6. J. Grandidier et al., "Light absorption enhancement in thin-film solar cells using whispering gallery modes in dielectric nanospheres," Adv. Mater. 23, 1272-1276 (2011).

7. C. R. Simovski et al., "Photovoltaic absorption enhancement in thin-film solar cells by nonresonant beam collimation by submicron dielectric particles," J. Appl. Phys. 114, 103104 (2013).

8. M. Schmid, P. Andrae, and P. Manley, "Plasmonic and photonic scattering and near fields of nanoparticles," Nanoscale Res. Lett. 9, 50 (2014).

9. Z. Chen, A. Taflove, and V. Backman, "Photonic nanojet enhancement of backscattering of light by nanoparticles: a potential novel visible-light ultramicroscopy technique," Opt. Express 12, 1214-1220 (2004).

10. S. Lee, L. Li, and Z. Wang, "Optical resonances in microsphere photonic nanojets," J. Opt. 16, 015704 (2014).

11. Y. E. Geints, E. K. Panina, and A. A. Zemlyanov, "Control over parameters of photonic nanojets of dielectric microspheres," Opt. Commun. 283(23), 4775-4781 (2010).

12. J. Yoon et al., "Ultrathin silicon solar microcells for semitransparent, mechanically flexible and microconcentrator module designs," Nat. Mater. 7(11), 907-915 (2008).

13. M. Paire et al., "Toward microscale $\mathrm{Cu}(\mathrm{In}, \mathrm{Ga}) \mathrm{Se} 2$ solar cells for efficient conversion and optimized material usage: theoretical evaluation," J. Appl. Phys. 108(3), 034907 (2010). 
14. P. Perez-Higueras et al., "High concentrator photovoltaics efficiencies: present status and forecast," Renewable Sustainable Energy Rev. 15, 1810-1815 (2011).

15. B. Reinhold et al., "Monolithically interconnected lameller $\mathrm{Cu}(\mathrm{In}, \mathrm{Ga}) \mathrm{Se} 2$ micro solar cells under full white light concentration," (2014), submitted for publication.

16. E. D. Palik, Handbook of Optical Constants of Solids, Academic Press, Orlando (1985).

17. M. Bass et al. Handbook of Optics, Third Edition Volume IV: Optical Properties of Materials, Nonlinear Optics, Quantum Optics, McGraw-Hill Education, NY (2009).

18. J. Hoffmann et al., "Comparison of electromagnetic field solvers for the 3-D analysis of plasmonic nano antennas," Proc. SPIE 7390, 73900J (2009).

19. www.jcmwave.com.

20. S. Burger et al., "Finite-element simulations of light propagation through circular subwavelength apertures," Proc. SPIE 7366, 736621 (2009).

21. www.zemax.com.

22. M. Schmid and P. Manley, "Enhancing solar cell conversion efficiency by lenses on the nano- and microscale," Proc. SPIE 9178, 91780K (2014).

23. M. Schmid et al., "Stability of plasmonic metal nanoparticles integrated in the back contact of ultra-thin $\mathrm{Cu}(\mathrm{In}, \mathrm{Ga}) \mathrm{S} 2$ solar cells," Thin Solid Films 527, 308-313 (2013).

24. C.-Y. Liu, "Ultra-high transmission of photonic nanojet induced modes in chains of coreshell microcylinders," Phys. Lett. A 376, 3261-3266 (2012).

Martina Schmid is a junior professor at the Freie Universität Berlin (FUB). She received her diploma in physics from the University of Augsburg in 2006, and her PhD from FUB in 2010. Since 2012, she has been leading a Helmholtz Young Investigator Group on "Nanooptical Concepts for Chalcopyrite Solar Cells" at the Helmholtz-Zentrum Berlin. Her current research interests include plasmonics and photonics for light management in ultra-thin solar cells as well as concentration concepts for micrometer-sized solar cells.

Phillip Manley is a final-year PhD student at the Helmholtz Zentrum Berlin. He received his master's degree in physics from the University of Durham in 2012. His current research interests include simulation of novel concepts in plasmonics and photonics with a particular focus on photovoltaics. 